\title{
O papel das redes de atenção na assistência a casos de violência por parceiro íntimo
}

\author{
The role of attention networks in assisting cases of violence by an intimate partner \\ El papel de las redes de atención en la asistencia en casos de violencia por un \\ compañero íntimo
}

\begin{abstract}
Marina Mussi Lima1*, Márcia Farsura de Oliveira ${ }^{1,2}$, Caroline Silva de Araujo Lima ${ }^{1}$, Marli do Carmo Cupertino ${ }^{1,3}$.
\end{abstract}

\section{RESUMO}

Objetivo: Revisar e verificar sobre as contribuições das Redes de Atenção (RA) à Violência por Parceiro Íntimo (VPI), na mudança no ciclo de agressões e melhoria da qualidade de vida da vítima de violência, no Brasil. Métodos: Foi feita uma revisão integrativa, usando como critério a busca nas bases de dados científicos SciELO e Acervo+, utilizando-se os descritores "Violência por Parceiro Íntimo AND Assistência Integral à Saúde" e "Violência contra a Mulher AND Assistência Integral a Saúde". Resultados: Observou-se que as RA à Saúde (RAS) e a RA a VPI (RAV) são facilitadores na abordagem pela Atenção Primária à Saúde (APS) às vítimas do sexo feminino. Demonstrou-se a importância do acolhimento e validação diagnóstica, cuidado, notificação e coordenação nas RAS e RAV dos casos de violência contra mulheres pela APS. As RAS e as RAV, no Brasil, podem contribuir para a mudança no ciclo de agressões, a partir do cuidado em saúde, proporcionado pelas equipes interdisciplinares. Considerações finais: As Redes de Atenção à VPI, coordenadas pela APS, podem atuar como promotoras da integralidade da usuária dos serviços de saúde do Sistema Único de Saúde (SUS).

Palavras-chave: Atenção primária à saúde, Violência de gênero, Assistência integral à saúde.

\begin{abstract}
Objective: To review and verify about the contributions of the Attention Networks (RA) to intimate partner violence (VPI), in changing the cycle of aggressions and improving the quality of life of the victim of violence, in Brazil. Methods: An integrative review was carried out, using as criteria the search in the scientific databases SciELO and Acervo+, using the descriptors "Violence by an Intimate Partner AND Comprehensive Health Care" and "Violence against Women AND Comprehensive Health Care". Results: It was observed that the RA to Health (RAS) and RA to VPI (RAV) are facilitators in the approach by Primary Health Care (APS) to female victims, demonstrating the importance of welcoming and diagnostic validation, care, notification and coordination in the RAS and RAV of cases of violence against women by APS. RAS and RAV, in Brazil, can contribute to the change in the cycle of aggressions, based on the health care provided by interdisciplinary teams. Final considerations: The VPI Care Networks, coordinated by the APS, can act as promoters of the integrality of the user of Health Unic System (SUS) health services.
\end{abstract}

Keywords: Primary health care, Gender violence, Comprehensive health care.

\footnotetext{
${ }^{1}$ Faculdade Dinâmica do Vale do Piranga (FADIP), Ponte Nova - MG. *E-mail: marina_mussi@hotmail.com

2 Universidade Federal de Juiz de Fora (UFJF), Juiz de Fora - MG.

3 Universidade Federal de Viçosa (UFV), Viçosa - MG.
}

SUBMETIDO EM: 6/2021

ACEITO EM: 6/2021

PUBLICADO EM: 7/2021 


\section{RESUMEN}

Objetivo: Revisión y verificar los aportes de las Redes de Atención (RA) a la violencia de pareja (VPI), en el cambio del ciclo de agresiones y mejorar la calidad de vida de la víctima de violencia, en Brasil. Métodos: Se realizó una revisión integradora, utilizando como criterio la búsqueda en las bases de datos científicas SciELO y Acervo +, utilizando los descriptores "Violencia por pareja íntima Y Atención Integral de Salud" y "Violencia contra la Mujer Y Atención Integral de Salud". Resultados: Se observó que la RA a la Salud (RAS) y RA a VPI (RAV) son facilitadores en el abordaje de Atención Primaria de Salud (APS) a mujeres víctimas, demostrando la importancia de la acogida y validación diagnóstica, atención, notificación y coordinación en el RAS y RAV de casos de violencia contra la mujer por APS. RAS y RAV, en Brasil, puede contribuir al cambio en el ciclo de las agresiones, con base en la atención de la salud, brindada por equipos interdisciplinarios.

Consideraciones finales: Las Redes de Atención a VPI, coordinadas por la APS, pueden actuar como promotoras de la integralidad del usuario de los servicios de salud del sistema único de Salud (SUS).

Palabras clave: Atención Primaria de Salud, Violencia de Género, Atención Integral de Salud.

\section{INTRODUÇÃO}

Segundo a Organização Mundial da Saúde (OMS) a Violência por Parceiro Íntimo (VPI) pode ser compreendida como a utilização da força física ou poder, em intimidação ou em atitude, auto ou hetero provocada, a indivíduos ou contra um grupo social, podendo provocar transtornos mentais, físicos ou orgânicos, desencadeando ou não incapacitação ou óbito à vítima (OMS, 2010).

A VPI é considerada uma violação explícita dos direitos humanos, tratando-se de um problema de saúde pública, no Brasil e no mundo. Possui impacto negativo na qualidade de vida e elevada taxa de morbimortalidade das pessoas agredidas, podendo envolver, além do sofredor, os familiares e amigos próximos (MASCARENHAS MDM, et al., 2020).

A nomenclatura estabelecida define que quando a agressão é feita a partir de um homem contra uma mulher, denomina-se "violência de gênero". E, na ocorrência de agressões promovidas pelo companheiro homem ou mulher - da vítima, denomina-se VPI (ROSA DOA, et al., 2018).

A VPI é vista em todas as classes sociais e vem desencadeando discussões, em busca de estabelecer estratégias para prevenção e, até mesmo, erradicação desse problema (ARBOIT J, et al., 2017). No Brasil, em $2011,70 \%$ dos registros de atendimentos realizados sobre violência feminina ocorreram na própria residência das vítimas (ROSA DOA, et al., 2018).

Nesse contexto, a Atenção Primária à Saúde (APS), que é o primeiro nível de atenção em saúde e a principal forma de acesso aos serviços do Sistema Único de Saúde (SUS), consiste na principal porta de entrada da vítima para a obtenção de suporte. Assim a APS possui papel imprescindível no manejo da atuação de prevenção e tratamento de base interdisciplinar, multiprofissional, intersetorial e socialmente engajada, desde o primeiro atendimento, no espaço físico de uma Unidade Básica de Saúde (UBS) (MINAYO MCS, 2007).

Adicionalmente a APS promove a detecção, o rastreio, o encaminhamento, as orientações à vítima e a notificação obrigatória, ou seja, o fluxo a ser seguido nas Redes de Atenção à Saúde (RAS) (TORRE DNPD, et al., 2016). Esse direcionamento da mulher atingida pelo ato violento tem como conceito a organização e articulação baseada na cooperação, reconhecimento e colaboração entre organizações, prezando pela autonomia e dinamismo dos setores envolvidos os quais englobam repartições públicas dentro e fora da área de saúde (MENDES EV, 2010).

A articulação entre esses setores modela as redes temáticas. Assim, as redes de atenção à VPI (RAV) são instituídas e dimensionadas a partir da realidade local de cada município, com a finalidade de garantir a integralidade da assistência à saúde e mudança no ciclo da violência (BRUHN MM e LARA L, 2016). 
Quanto às ocorrências de agressões, infere-se por descrições na literatura, que provocam mais mortes em mulheres de 15 a 44 anos, do que o câncer, a malária, os acidentes de trânsito e até mesmo guerras (ROSA DOA, et al., 2018). Além dessas informações, observa-se que o índice de registros de atendimentos necessários, em decorrência de eventos ofensivos contra pessoas do sexo feminino, de acordo com os Dados da Central de Atendimento à Mulher/ Ligue 180, entre 2005 e 2015, corresponderam a 4.708.978 no Brasil (SANTOS WJ, et al., 2014). Desses, foram feitos 223.796 registros, em 2015, tendo como causa principal a violência: (i) doméstica; (ii) sexual; e (iii) outras. Além disso, duas em cada três dessas vítimas de violência (147.691) eram mulheres (SANTOS WJ, et al., 2014).

Dessa forma, atentando para os dados apresentados quanto à relevância desse problema social e de saúde pública, que pode ocasionar implicações mentais e física das vítimas, objetivou-se verificar, na literatura científica, quais as contribuições das Redes de Atenção à VPI, coordenada pela APS, na mudança no ciclo de agressões, e consequente contribuição para a melhoria da qualidade de vida da mulher vítima de violência, no Brasil.

\section{MÉTODOS}

Trata-se de uma revisão integrativa, na qual foi feita uma busca por estudos brasileiros publicados, na forma de artigo, entre os anos de 2016 e 2021. Como critérios de inclusão, buscou-se artigos que tratavam das temáticas: I) Redes de Atenção à VPI; II) APS associada à VPI; e III) Redes Públicas Inter setoriais associada a VPI, publicados em língua portuguesa e disponíveis na forma de texto completo.

Como critérios de exclusão foram considerados: I) teses, dissertações, monografias e artigos de revisão ou com texto completo indisponível; II) artigos publicados anteriormente a 2016; III) Estudos que não foram feitos no Brasil; IV) estudos feitos no Brasil, mas que não foram publicados em língua portuguesa; e V) estudos que abordavam atenção primária ou redes públicas inter setoriais não associados a redes de atenção a VPI. Após aplicação dos critérios de inclusão e exclusão, foram selecionados 11 artigos. O pequeno número de publicações, especificamente sobre o assunto, foi uma limitação para este estudo. Grande número de pesquisas foram excluídas, as quais tratavam a temática sem a menção tácita ao papel das redes de atenção na assistência a casos de VPI.

A pesquisa foi realizada no período de março de 2020 a abril de 2021, utilizando-se os descritores em ciências da saúde (DeCS): "Violência por Parceiro Íntimo AND Assistência Integral à Saúde" e "Violência contra a Mulher AND Assistência Integral à Saúde", nas bases de dados on-line Acervo+ [acervomais.com] e Scientific Electronic Library Online (SciELO) [com os filtros "Brasil", e publicações a partir de 2016, até março de 2021, no idioma "português" e "artigo"].

Baseando-se nos critérios de inclusão e de exclusão pré-regulamentados, foi feita a seleção dos artigos a partir da leitura de: (i) título; (ii) resumo; e (iii) palavras-chaves. Os resultados foram expostos após leitura e interpretação dos textos completos com compilação dos dados e informações obtidas.

Ademais, a Lei 11.340, a qual é também denominada "Lei Maria da Penha" foi utilizada como referencial teórico para o presente trabalho, já que a mesma salienta o enfoque na gravidade da violência doméstica contra pessoas do sexo feminino, considerando 5 tipos de agressão: física, psicológica, sexual, patrimonial e moral (BRASIL, 2006).

\section{RESULTADOS E DISCUSSÃO}

Foram recuperados 1086 artigos, na base de dados SciELO, e 279 artigos, na base de dados Acervo+, com a utilização dos filtros. Com tal procedimento foram obtidos 1365 artigos. Depois da aplicação dos critérios de inclusão e exclusão restaram 11 artigos, que foram incluídos neste estudo, conforme está descrito no fluxograma Prisma da Figura 1.

Os dados sobre o objetivo, tipo de rede de assistência, população estudada, método de coleta de dados e resultados principais dos artigos incluídos no estudo, encontram-se apresentados no Quadro 1. 
Figura 1 - Diagrama de fluxo dos resultados da pesquisa de literatura com base na declaração do PRISMA.

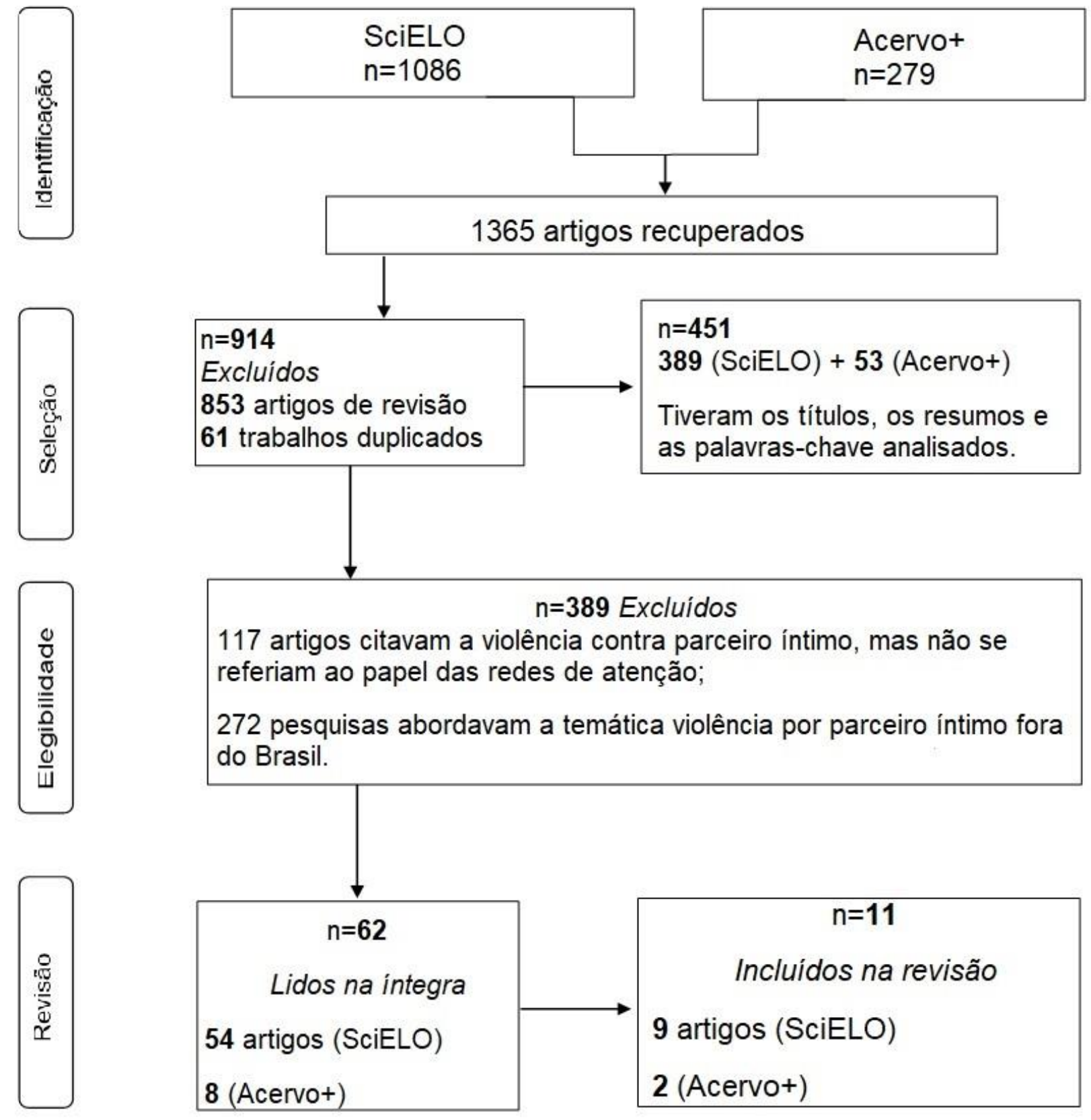

Fonte: Lima MM, et al., 2021. 


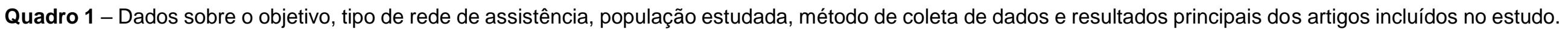

\begin{tabular}{|c|c|c|c|c|c|}
\hline Autor e ano & Objetivos do estudo & Rede & Amostra & Método & Conclusão \\
\hline $\begin{array}{l}\text { LEITE AC e } \\
\text { FONTANELLA } \\
\text { BJB, } 2019 .\end{array}$ & $\begin{array}{l}\text { Compreender as dificuldades } \\
\text { subjetivas de notificar a violência } \\
\text { doméstica por profissionais da } \\
\text { atenção primária. }\end{array}$ & RAV e RAS & $\begin{array}{l}14 \text { profissionais da } \\
\text { atenção primária. }\end{array}$ & $\begin{array}{l}\text { Entrevista semi } \\
\text { estruturada }\end{array}$ & $\begin{array}{l}\text { Falta de conhecimento dos meios de notificação; } \\
\text { serviços de saúde inapropriados para notificar; } \\
\text { boletim de ocorrência policial como principal } \\
\text { instrumento de notificação; a notificação seria } \\
\text { opcional; e a burocracia excessiva. }\end{array}$ \\
\hline $\begin{array}{l}\text { FIGUEIREDO, } \\
\text { IATS, et al., } \\
2020 \text {. }\end{array}$ & $\begin{array}{l}\text { Descrever o conhecimento sobre a } \\
\text { violência contra a mulher das } \\
\text { discentes de medicina de uma } \\
\text { instituição de ensino do Pará. }\end{array}$ & RAV e RAS & $\begin{array}{l}141 \text { discentes de } \\
\text { medicina }\end{array}$ & Questionário individual & $\begin{array}{l}56 \% \text { responderam que a mulher não é bem tratada } \\
\text { no país, } 89 \% \text { responderam que a mulher não é } \\
\text { tratada de forma igual ao homem. }\end{array}$ \\
\hline $\begin{array}{l}\text { DEFILIPO EC, et } \\
\text { al., } 2020 .\end{array}$ & $\begin{array}{l}\text { Caracterizar a violência na gestação e } \\
\text { verificar a associação com os fatores } \\
\text { socioeconômicos, demográficos, } \\
\text { obstétricos, comportamentais, de } \\
\text { assistência à saúde e doenças na } \\
\text { gestação. }\end{array}$ & RAV e RAS & Puérperas & $\begin{array}{l}\text { Entrevista, e } \\
\text { informações do cartão } \\
\text { de pré-natal e } \\
\text { prontuário. }\end{array}$ & $\begin{array}{l}\text { Dessas, } 62(8,0 \%) \text { relataram ter sofrido violência } \\
\text { física, psicológica ou sexual durante a gestação. As } \\
\text { gestantes com maior chance de terem sofrido } \\
\text { violência foram as dependentes de álcool, as que } \\
\text { não realizaram o pré-natal, as que usaram os } \\
\text { serviços de saúde de forma emergencial durante a } \\
\text { gravidez e que apresentaram diabetes gestacional } \\
\text { e DST. }\end{array}$ \\
\hline $\begin{array}{l}\text { PEREIRA VOM, } \\
\text { et al., } 2020 .\end{array}$ & $\begin{array}{l}\text { Caracterizar o perfil das violências, } \\
\text { das vítimas e dos prováveis autores } \\
\text { das violências contra adolescentes. }\end{array}$ & RAV e RAS & Adolescentes & $\begin{array}{l}\text { Registros no Sistema } \\
\text { de Informação de } \\
\text { Agravos de Notificação }\end{array}$ & $\begin{array}{l}\text { As notificações de } 75,4 \% \text { dos municípios } \\
\text { brasileiros. A violência física predominou no sexo } \\
\text { masculino ( } 15 \text { a } 19 \text { anos), e psicológica no sexo } \\
\text { feminino ( } 10 \text { e } 14 \text { anos. A violência sexual } \\
\text { prevaleceu no sexo feminino, entre } 10 \text { e } 14 \text { anos, } \\
\text { nos indígenas, negros e amarela. A negligência } \\
\text { predominou no sexo masculino ( } 0 \text { e } 14 \text { anos). } \\
\text { Ambos no domicílio, por agressores familiares. }\end{array}$ \\
\hline $\begin{array}{l}\text { MASCARENHAS } \\
\text { MDM, et al., } \\
2020 .\end{array}$ & $\begin{array}{l}\text { Analisar as notificações de violência } \\
\text { por parceiro íntimo contra mulheres. }\end{array}$ & RAV & $\begin{array}{l}\text { Mulheres }(\geq 15 \\
\text { anos). }\end{array}$ & $\begin{array}{l}\text { Dados do Sistema de } \\
\text { Informação de Agravos } \\
\text { de Notificação }\end{array}$ & $\begin{array}{l}\text { Foram notificados } 454.984 \text { casos de violência } \\
\text { perpetrados por homens contra mulheres, dos quais } \\
62,4 \% \text { eram VPI. Sendo: abusos físicos }(86,6 \%) \text {, } \\
\text { psicológicos }(53,1 \%) \text { e sexuais }(4,8 \%) \text {. }\end{array}$ \\
\hline
\end{tabular}




\begin{tabular}{|c|c|c|c|c|c|}
\hline Autor e ano & Objetivos do estudo & Rede & Amostra & Método & Conclusão \\
\hline $\begin{array}{l}\text { BRANCO JGO, } \\
\text { et al., } 2020 .\end{array}$ & $\begin{array}{l}\text { Identificar as condições que } \\
\text { comprometem a atenção à pessoas } \\
\text { vítimas de VPI e à estruturação da } \\
\text { rede. }\end{array}$ & RAS & $\begin{array}{l}19 \text { gestores e } \\
\text { trabalhadores que } \\
\text { assistem mulheres } \\
\text { em situação de } \\
\text { violência sexual }\end{array}$ & $\begin{array}{l}\text { Entrevistas } \\
\text { semi estruturadas }\end{array}$ & $\begin{array}{l}\text { O cotidiano dos serviços é permeado pela redução } \\
\text { do número e rotatividade de profissionais, precárias } \\
\text { condições de trabalho, inadequação da estrutura } \\
\text { física para o desenvolvimento da atenção, além das } \\
\text { lacunas na capacitação e sensibilização. }\end{array}$ \\
\hline $\begin{array}{l}\text { MOREIRA KFA, } \\
\text { et al., } 2020 .\end{array}$ & $\begin{array}{l}\text { Descrever o perfil das mulheres que } \\
\text { sofreram violência sexual, bem como } \\
\text { identificar as características do } \\
\text { agressor e da violência sexual. }\end{array}$ & RAV e RAS & $\begin{array}{l}\text { Mulheres em idade } \\
\text { fértil }\end{array}$ & $\begin{array}{l}\text { Dados do Sistema de } \\
\text { Informação de Agravos } \\
\text { de Notificação }\end{array}$ & $\begin{array}{l}\text { Houve } 327 \text { casos de violência sexual, na maioria } \\
\text { entre } 10 \text { a } 19 \text { anos }(83,18 \%) \text {. Parda }(54,74 \%) \text {, } \\
\text { solteiras }(77,06 \%), \leq 8 \text { anos de estudo }(49,85 \%) \text {. A } \\
\text { agressão ocorreu a noite }(23,86 \%) \text {, na residência } \\
\text { (59,33\%), por conhecidos }(81,04 \%) \text {. } 72,17 \% \text { foram } \\
\text { atendidas nos serviços de saúde, com prescrição de } \\
\text { profilaxia para DST }(45,8 \%)\end{array}$ \\
\hline $\begin{array}{l}\text { NETO KREM e } \\
\text { GIRIANELI VR, } \\
\quad 2020 .\end{array}$ & $\begin{array}{l}\text { Descrever as características da } \\
\text { violência contra as mulheres } \\
\text { notificadas no município de São } \\
\text { Paulo, 2008-2015. }\end{array}$ & RAS & $\begin{array}{l}\text { Mulheres (20 e } 59 \\
\text { anos ) vítimas de } \\
\text { violência } \\
\text { interpessoal. }\end{array}$ & $\begin{array}{l}\text { Dados do Sistema de } \\
\text { Informação e Vigilância } \\
\text { de Violências e } \\
\text { Acidentes da } \\
\text { Coordenação em } \\
\text { Vigilância em Saúde }\end{array}$ & $\begin{array}{l}3.256 \text { casos. A violência física predominou ( } 81,3 \%) \\
\text { entre mulheres ( } 20 \text { a } 29 \text { anos- } 37,6 \%) \text {. O agressor } \\
\text { do sexo masculino }(47,7 \%) \text { e conhecido }(62,5 \%) \text {, na } \\
\text { residência }(31,6 \%) \text {; Partes mais atingidas: cabeça } \\
(31 \%) \text { e membros }(21 \%) \text {, gerando traumas }(60 \%) \text {. }\end{array}$ \\
\hline $\begin{array}{l}\text { MELLO MMP, et } \\
\text { al., } 2021 .\end{array}$ & $\begin{array}{l}\text { Entender as equipes } \\
\text { multidisciplinares atuantes em } \\
\text { Juizados de violência contra a mulher. }\end{array}$ & RAV e RAS & $\begin{array}{l}\text { Equipes } \\
\text { multidisciplinares } \\
\text { para os Juizados }\end{array}$ & $\begin{array}{l}\text { Falas e reflexões } \\
\text { extraídas dos grupos } \\
\text { focais e entrevistas }\end{array}$ & $\begin{array}{l}\text { As equipes multidisciplinares buscam novas saídas } \\
\text { à violência doméstica contra a mulher no Brasil. }\end{array}$ \\
\hline $\begin{array}{l}\text { CONCEIÇÃO, } \\
\text { HN, et al., } 2021\end{array}$ & $\begin{array}{l}\text { Analisar a prevalência e fatores } \\
\text { associados à violência por parceiro } \\
\text { íntimo na gestação. }\end{array}$ & RAV & $\begin{array}{l}\text { Grávidas de } 10 \text { a } 49 \\
\text { anos de idade, no } \\
\text { terceiro trimestre }\end{array}$ & $\begin{array}{l}\text { Entrevistas e análise } \\
\text { hierarquizada por } \\
\text { regressão logística } \\
\text { múltipla. }\end{array}$ & $\begin{array}{l}\text { A violência na gestação prevaleceu em } 33 \% \text { com } \\
\text { predomínio psicológico }(18,9 \%) \text {. A faixa etária da } \\
\text { mulher }<20 \text { anos e o consumo de drogas ilícitas pelo } \\
\text { parceiro associados a violência. }\end{array}$ \\
\hline $\begin{array}{l}\text { SOUZA TMC e } \\
\text { MARTINS TF, } \\
2021\end{array}$ & $\begin{array}{l}\text { Conhecer as vivências de uma rede } \\
\text { intersetorial na investigação de } \\
\text { concepções de violência doméstica e } \\
\text { o cotidiano de trabalho no } \\
\text { atendimento às mulheres. }\end{array}$ & RAV & $\begin{array}{l}\text { Quatro policiais: } 2 \\
\text { do sexo feminino e } 2 \\
\text { sexo masculino }\end{array}$ & $\begin{array}{c}\text { Entrevistas semi } \\
\text { estruturadas realizadas }\end{array}$ & $\begin{array}{l}\text { Falta de: espaço físico, funcionários, tempo e } \\
\text { capacitação foram apontados como dificuldades } \\
\text { para a realização de atendimento adequado às } \\
\text { mulheres. O contato diário com a violência provoca } \\
\text { a naturalização, interferindo na qualidade do } \\
\text { trabalho. }\end{array}$ \\
\hline
\end{tabular}

Legenda: Redes de Atenção à Saúde (RAS), Redes de Atenção à Violência por Parceiro Íntimo (RAV).

Fonte: Lima MM, et al., 2021. 
Dos 11 artigos incluídos na pesquisa, 6 tratavam das duas redes de atenção (RAV e RAS); 3 artigos tratavam apenas do papel da RAV; 2 artigos apenas tratavam sobre o papel da RAS. Quanto às características gerais, a produção investigada que teve a data de publicação mais antiga foi a de Leite AC e Fontanella BJB (2019) e a mais recente foi a de Souza TMC e Martins TF (2021).

De acordo com a característica da amostra, 4 artigos tinham como população estudada os profissionais que atendiam às vítimas de violência, amostra sendo composta por profissionais de saúde, equipes interdisciplinares e policiais (LEITE AC e FONTANELLA BJB, 2019; BRANCO JGO, et al., 2020; MELLO MMP, et al., 2021; SOUZA TMC e MARTINS TF, 2021). Outros 5 tratavam especificamente de vítimas mulheres, 2 deles abordando mulheres em período gravídico puerperal (DEFILIPO EC, et al., 2020; MASCARENHAS MDM, et al., 2020; MOREIRA KFA, et al., 2020; NETO KREM e GIRIANELLI VR, 2020; CONCEIÇÃO HN, et al., 2021). Um artigo teve como público os adolescentes e outo trabalho abordou acadêmicos de medicina (FIGUEIREDO IATS, et al., 2020; PEREIRA VOM, et al., 2020).

Em relação ao método de coleta de dados, a maioria dos estudos utilizaram-se de entrevistas com os participantes (LEITE AC e FONTANELLA BJB, 2019; BRANCO JGO, et al., 2020; CONCEIÇÃO HN, et al., 2021; MELLO MMP, et al., 2021; SOUZA TMC e MARTINS TF, 2021). A outra parte, somando-se 4 artigos, utilizou o método de coleta de dados dos sites de notificação (FIGUEIREDO IATS, et al., 2020; MASCARENHAS MDM, et al., 2020; MOREIRA KFA, et al., 2020; NETO KREM e GIRIANELLI VR, 2020; PEREIRA VOM, et al., 2020).

Outro estudo realizado também com dados do SINAN, concluiu que as agressões às mulheres, em diferentes fases da vida e pertencentes aos distintos grupos sociais, consistem em agravantes de transtornos mentais e comportamentais, justificando o fato de tal temática compor a agenda de políticas públicas, para que se garanta a integralidade das vítimas conforme o que é preconizado pelo SUS, minimizando o determinante social da doença violência (MOREIRA KFA, et al., 2020).

Um estudo realizado em São Paulo, com mulheres com idade entre 20 e 59, trouxe como desfecho que as subnotificações ainda são frequentes, apesar do número de casos de violência física no município ter aumentado, sugerindo que um maior volume de informações sobre as ocorrências de atos violentos contra pessoas do sexo feminino tem chegado aos órgãos competentes (NETO KREM E GIRIANELLI VR, 2020).

Um estudo realizado com puérperas que deram à luz em um Hospital na cidade de Governador Valadares, revelou que a violência contra a mulher gestante ocorre tanto em ambiente domiciliar, quanto fora, sem que, na grande maioria dos casos, os profissionais de saúde assistentes não fazem o levantamento dessa hipótese, durante os atendimentos. Por isso, é importante que sejam realizadas ações em saúde que orientem o rastreio e o reconhecimento dos sinais e dos sintomas comumente apresentados pelas vítimas, o mais precocemente possível, evitando desfechos negativos (DEFILIPO EC, et al., 2020).

Um estudo realizado com estudantes de medicina do estado do Pará, trouxe à tona que as participantes revelaram possuir familiaridade com eventos de agressões a mulheres, demonstrando que tal brutalidade não tem predileção por nenhum grupo social específico (FIGUEIREDO IATS, et al., 2020). Outro estudo, conduzido com profissionais da atenção primária conclui que a violência contra a mulher (VCM) necessita ser tratada pela atenção básica à saúde, porém os profissionais da atenção básica à saúde apresentam dúvidas sobre as melhores condutas a serem adotadas (LEITE AC e FONTANELLA BJB, 2019).

Em estudo realizado com gestores e profissionais da saúde, concluiu-se que os trabalhadores e gestores precisam se fixar por períodos maiores nas equipes de APS, a fim de garantir a longitudinalidade do cuidado, com aumento do vínculo entre as usuárias dos serviços de saúde e seus respectivos profissionais, facilitando a presunção diagnóstica e o fomento à atenção integral à saúde da mulher, incluindo os programas de planejamento familiar (BRANCO JGO, et al., 2020).

O desfecho de um estudo realizado com policiais civis de Jataí - Goiás, confirmou que a sociedade apresenta concepções de direitos e de deveres distintas e de forma desigual, entre as mulheres e os homens, o que pode ser percebido, inclusive, nos serviços que compõem as RAS e as RAV, levando à necessidade 
iminente de alterações desse panorama, a fim de se atingir a interrupção do preconceito e do ciclo vicioso de VCM, para que a mesma possa ser acolhida e tratada em conformidade com os preceitos de humanização e da bioética (SOUZA TMC e MARTINS TF, 2021).

Um estudo multicêntrico, envolvendo Equipes multidisciplinares de Juizados de Violência Doméstica e Familiar contra a Mulher, concluiu que é necessário enxergar/admitir as rígidas fronteiras do "mundo jurídico" (MELLO MMP, et al., 2021). Após a execução de uma pesquisa por meio do SINAN, os autores Pereira VOM, et al. (2020) concluíram que devido os problemas serão complexos, os mesmos demandam atuação intersetorial para seu enfrentamento.

Historicamente, a evolução dos direitos humanos abrange os direitos de primeira geração, referidos nas Revoluções americana e francesa, compreendendo liberdades individuais, como a de consciência, de reunião e a inviolabilidade de domicílio. Os direitos sociais ou direitos de segunda geração, são aqueles que dizem respeito a assistência social, saúde, educação, trabalho, lazer etc., realçando também o princípio da igualdade. Já nos chamados de terceira geração, pertence à titularidade coletiva, como direito à paz, ao desenvolvimento, à qualidade do meio ambiente, à conservação do patrimônio histórico e cultural (NETO KREM e GIRIANELLI VR, 2020).

Diante dessa perspectiva, através da Declaração Universal dos Direitos Humanos (DUDH) da Organização das Nações Unidas (ONU), os direitos fundamentais são de caráter universal, inalienáveis, invioláveis, interdependentes, inter-relacionados, sem relação com classe social, orientação sexual, renda, cultura, escolaridade, idade e religião. Reconhecidos mundialmente por meio de pactos e tratados, os direitos nascem com o indivíduo, conferem proteção e respeito à sua dignidade, à vida, à liberdade, e à igualdade (NETO KREM e GIRIANELLI VR, 2020).

De acordo com o Escritório das Nações Unidas sobre Drogas e Crime, mundialmente, a violência de gênero não tem sido observada verdadeiramente como uma infração aos direitos humanos, sendo que apenas em 52 países existe legislação voltada às ocorrências de relações sexuais forçadas ou outras coerções conjugais praticadas pelo parceiro íntimo da pessoa do sexo feminino, sem o seu tácito consentimento (UNODC, 2020; MELLO MMP, et al., 2021).

No Brasil, a Lei Maria da Penha número 11.340 de 2006, como instrumento de política pública, ampara todo ato praticado contra as mulheres no contexto doméstico, familiar ou de qualquer relação íntima de afeto. Ainda assim, encontra resistência na efetivação seja por parte da sociedade ou do Poder Público (CONCEIÇÃO HN, et al., 2021). Com o advento e vigência da Lei, gozando o objetivo de prevenir, punir e erradicar essa epidemia global de saúde, permitiu-se a identificação com maior lucidez, da violação dos direitos fundamentais no que tange a mulher, devido à violência de sua integridade física e psicológica (CONCEIÇÃO HN, et al., 2021; MELLO MMP, et al., 2021).

As RAS e as RAV, na abordagem às vítimas do sexo feminino que sofreram VPI, têm obtido resultados positivos na alteração no curso de agressões, de acordo com dados identificados pela presente pesquisa, quando: (i) a mulher vítima de violência é atendida pela equipe de APS e conduzida e coordenada por esta última dentro das RAS e das RAV; (ii) há a suspeição e a confirmação do diagnóstico da VCM; (iii) ocorrem capacitações voltadas à educação permanente em saúde para o enfrentamento de VPI; e (iv) existe envolvimento da sociedade com a causa, requerendo maior controle social e vigilância das ações intrassetoriais e intersetorias integradas nas RAS e nas RAV como, por exemplo, a notificação ao Sistema de Informações de Agravos de Notificação (SINAN), conforme descrito a seguir (BRANCO JGO, et al., 2020; FIGUEIREDO IATS, et al., 2020; NETO KREM e GIRIANELLI VR, 2020; PEREIRA VOM, et al., 2020; CONCEIÇÃO HN, et al., 2021; SOUZA TMC e MARTINS TF, 2021):

A capacitação permanente, formação continuada dos profissionais de saúde e atenção da equipe de saúde à mulher vítima de violência, relacionam-se, à medida que estudos apontam que mulheres vítimas de violência são frequentadoras assíduas em serviços de saúde, principalmente quando comparadas àquelas que não se encontram nessa circunstância (BRANCO JGO, et al., 2020). Assim, torna-se evidente a necessidade de 
atendimento integral pela equipe interdisciplinar de caráter intersetorial com a não segmentação das ações como previsto na Lei Maria da Penha nas UBS (LEITE AC e FONTANELLA BJB, 2019; DEFILIPO EC, et al., 2020).

Além disso, para os exercícios profissionais na área de saúde, existem os códigos de ética, que são conjuntos de princípios morais e normas que são entendidos como deveres e obrigações a serem realizados, assim, em uma equipe interdisciplinar, cada profissional deve se basear no código de ética para adotar condutas ao longo do seu trabalho. Esses princípios estabelecidos não apresentam explicitamente a notificação da violência como um dever do profissional, entretanto, julga obrigatório o zelo pela saúde, dignidade e integridade humana, promovendo qualidade de vida e respeitando os direitos humanos (PEREIRA VOM, et al., 2020).

Nesse contexto, cabe ressaltar a correlação entre o desconhecimento da epidemiologia da VCM por profissionais da área da saúde com a subnotificação dos casos, não permitindo reflexão, discussões de casos e procedimentos padrão de referência e contrarreferência quanto a esse problema social e de saúde pública. Estudos mostram a falha de comunicação na relação médico-paciente. Sendo assim, sabe-se da importância deontológica da equipe para promoção de atendimento mais humanizado e individualizado, visando o bemestar geral da paciente, ao fomentar a obrigatoriedade da notificação compulsória de caráter sigiloso em todo em território nacional como previsto pelo Ministério da Saúde Lei no 10.778 de 2003 Portaria GM/MS no 104, de 25 de janeiro de 2011 (BRANCO JGO, et al., 2020).

Ademais, no SUS, as determinações da Lei Maria da Penha precisam e devem ser reconhecidas e aplicadas, assim como nos demais serviços de saúde, proporcionando uma mudança na perspectiva popular, ao ser analisada como problema social de fato. Através da mobilização social, essas e outras formas de políticas públicas vêm sendo implementadas no contexto do fim da VCM (NETO KREM e GIRIANELLI VR, 2020). Além da Lei Maria da Penha, em 2015 o feminicídio, após uma alteração do artigo 121 do Código Penal pela Lei no 13.104/2015, passou a ser qualificado como crime hediondo no Brasil e a tipificação penal do estupro em 2009. Também como a criação de serviços especializados como a Central de Atendimento à Mulher - Ligue 180, oferecido gratuitamente pela Ouvidoria Nacional dos Direitos Humanos garantem ampliação do acesso aos direitos (BRASIL, 2006; MASCARENHAS MDM, et al., 2020).

Cabe ressaltar a criação de uma rede de atenção articulada e intersetorial, prevendo assistência integral à mulher no contexto de violência, conforme os princípios e diretrizes previstos na Lei Orgânica da Assistência Social, no Sistema Único de Saúde (SUS) e a importância da equipe interdisciplinar e multiprofissional na APS engajada desde o primeiro atendimento à vítima (BRASIL, 1993; LEITE AC e FONTANELLA BJB, 2019).

O diagnóstico da VCM requer investigação ativa por parte dos profissionais de saúde, acolhimento adequado, com ações almejando a detecção dos casos, como por exemplo a instituição de canais de comunicação competentes, oferta de testes anti-HIV, aconselhamento das vítimas e reestruturação da assistência à saúde interligando a saúde e os direitos humanos. Estudos mostram também a necessidade de preparação da equipe interdisciplinar, através de cursos de capacitação e de reciclagem de conhecimentos, para realizarem a abordagem específica e gerar visibilidade desse problema social (MASCARENHAS MDM, et al., 2020).

É visto que há relevante prevalência de uso dos serviços de saúde e que esse último aumenta com a intensidade, ocorrência e gravidade da VPI. Mesmo após cessar os atos de violência, as mulheres apresentam maiores taxas de consultas, em sua maioria, devido ao acompanhamento da saúde mental, uma vez que as vítimas retratam tanto efeitos agudos, quanto impactos a longo prazo, como dores crônicas, problemas gastrintestinais, infecções sexualmente transmissíveis, infecções urinárias de repetição e alterações menstruais e sexuais (FIGUEIREDO IATS, et al., 2020).

Cabe ressaltar ainda, que estudos apontam a maior incidência de VPI entre mulheres com maior número de filhos, além de menor uso de preservativos nas relações sexuais, aumentando o risco de infecção por doenças sexualmente transmissíveis; e a violência moderada a grave está mais associada à infecção pelo vírus HIV e revelação do diagnóstico ao parceiro (MOREIRA KFA, et al., 2020). 
Nesse contexto, é válido ressaltar que a vigilância das ações intrasetoriais e intersetoriais tem papel fundamental para assegurar ações e políticas efetivas, preventivas e permanentes contra a VPI e capazes de responder às demandas inerentes da parcela da sociedade acometida. Conseguem, dessa forma, garantir 0 atendimento interdisciplinar qualificado, planejamento ativo das ações já estabelecidas e de futuras ideias, além de analisar a utilização do orçamento de verbas federais, estaduais e municipais destinadas às problemáticas relativas à VPI. As RAS entram nesse contexto, a partir do momento que, por intermédio da articulação entre poder público e a sociedade civil, podem avaliar e controlar os serviços oferecidos (LEITE AC e FONTANELLA BJB, 2019).

Ainda outro fator que vale ressaltar é a efetivação de práticas do MS, por meio da APS, ao desenvolver serviços humanizados e promoção da cultura da paz. Assume-se com a população então, o compromisso e responsabilidade de prevenção da VPI. Faz-se necessário ainda, a vigilância desses papéis, para reafirmar a universalização do acesso e os direitos das mulheres independente de diferenças de raça, orientação sexual e cultura. Sustenta a integralidade do acolhimento à vítima, desde o primeiro atendimento e suspeita de violência, até o engajamento da segurança e justiça pública. Essas ações também podem estar envolvidas com a divulgação de informações pela mídia, atividades educativas e criação de fóruns e comissões, para que assim haja o fortalecimento da escuta (MOREIRA KFA, et al., 2020).

\section{CONSIDERAÇÕES FINAIS}

O estudo mostrou que é necessário promover a integralidade do usuário pelo princípio doutrinário do SUS. Entretanto, para que isso ocorra de forma efetiva, as gestões responsáveis, precisam incluir pautas que atendam às práticas cotidianas de cuidado ao indivíduo em situação de vulnerabilidade, por meio: de políticas públicas e de atividades de educação permanente nas redes intrassetoriais e intersetoriais. As RAS e as RAV, no Brasil, contribuem para a mudança no ciclo de agressões, através das ações de saúde, das equipes interdisciplinares devido ao acolhimento, coordenação e direcionamento do fluxo entre os serviços, favorecendo a redução dos impactos na saúde mental dos indivíduos agredidos.

\section{REFERÊNCIAS}

1. ARBOIT J, et al. Atenção à saúde de mulheres em situação de violência: desarticulação dos profissionais em rede. Rev. esc. enferm. USP, 2017; 51: e03207.

2. BARBOSA SN. A participação brasileira na construção da Declaração Universal sobre Bioética e Direitos Humanos da UNESCO. Revista Brasileira de Bioética, 2006; 2(4): 423-436.

3. BERNARDES MG. Ética em pesquisas com seres humanos: origem e documentação reguladora. Escola de Saúde do Exército. Rio de Janeiro, 2008.

4. BRANCO JGO, et al. Fragilidades no processo de trabalho na Atenção à Saúde à Mulher em situação de violência sexual. Ciênc. saúde coletiva, Rio de Janeiro, 2020; 25(5): 1877-1886.

5. BRASIL. Presidência da República. Casa Civil. Subchefia para Assuntos Jurídicos. Lei no 11.340, de 7 de agosto de 2006. Cria mecanismos para coibir e prevenir a violência doméstica e familiar contra a mulher e dá outras providências. 2006. Disponível em: http://www.planalto.gov.br/ccivil_03/_Ato2004-2006/2006/Lei/L11340.html Acesso em: 13 mar. 2021.

6. BRASIL. Ministério do Desenvolvimento Social e Combate à Fome. Secretaria Nacional de Assistência Social. Lei Orgânica de Assistência Social - Lei $\mathrm{n}^{0}$ 8742, de 7 de dezembro de 1993 . Disponível em: http://www.planalto.gov.br/ccivil_03/leis//8742compilado.html Acesso em 16 jul. 2021.

7. BRUHN MM e LARA L. Rota crítica: a trajetória de uma mulher para romper o ciclo da violência doméstica. Rev. Polis Psique, Porto Alegre, 2016; 6(2): 70-86.

8. CAVALCANTI SVSF. A violência doméstica como violação dos direitos humanos. Revista Jus Navigandi, Teresina, 2005; 10(901): 12-26.

9. CONCEIÇÃO HN, et al. Prevalência e fatores associados à violência por parceiro íntimo na gestação em Caxias, Maranhão, 2019-2020. Epidemiol Serv Saúde, 2021.

10. DEFILIPO EC, et al. Violência contra a gestante e fatores associados no município de Governador Valadares. Rev Saúde Pública. 2020.

11. FIGUEIREDO IATS, et al. Conhecimento sobre violência contra a mulher pelas discentes do curso de medicina de uma instituição do Pará. Revista Artigos. Com, 2020; 19: e4007.

12. LEITE AC e FONTANELLA BJB. Violência doméstica contra a mulher e os profissionais da APS: Predisposição para abordagem e dificuldades com a notificação. Revista Brasileira de Medicina de Família e Comunidade, 2019; 14(41): 2059. 
13. MARINHO PAM e GONÇALVES HS. Práticas de empoderamento feminino na América Latina. Rev. Estud. Soc, Bogotá, 2016; 56: 80-90.

14. MASCARENHAS MDM, et al. Análise das notificações de violência por parceiro íntimo contra mulheres, Brasil, 20112017. Revista Brasileira de Epidemiologia, 2020; 23: 1-13.

15. MELLO MMP, et al. Para além do "mundo jurídico": um diálogo com as equipes multidisciplinares de Juizados (ou Varas) de Violência Doméstica. Rev. Direito e Práx., Rio de Janeiro, 2021; 12(01): 608-641.

16. MENDES EV. As redes de atenção à saúde. Ciênc. saúde coletiva, Rio de Janeiro, 2010; 15(5): $2297-2305$.

17. MINAYO MCS, et al. Institucionalização do tema da violência no SUS: avanços e desafios. Ciênc. saúde coletiva, Rio de Janeiro, 2018; 23(6): 2007-2016.

18. MOREIRA KFA, et al. Violência sexual contra mulheres em idade fértil na região norte do Brasil. Revista Eletrônica Acervo Saúde, 2020; 12(3): e2826.

19. NETO KREM e GIRIANELLI VR. Evolução da notificação de violência contra mulher no município de São Paulo, 20082015. Cad. saúde colet., Rio de Janeiro, 2020; 28(4): 488-499.

20. ORGANIZAÇÃO MUNDIAL DA SAÚDE (OMS). Prevenção da violência sexual e da violência pelo parceiro íntimo contra a mulher: ação e produção de evidência Washington: OMS; 2010. Disponível em: http://apps.who.int/iris/bitstream/handle/10665/44350/9789275716359_por.pdf;jsessionid=E778CC8FFCB124916B39 BA568A58C5DC?sequence=3 Acesso em 16 jul. 2021.

21. PEREIRA VOM, et al. Violências contra adolescentes: análise das notificações realizadas no setor saúde, Brasil, 2011-2017. Rev Bras Epidemiol 2020; 23: E200004.Supl.1.

22. PINHEIRO R. Integralidade. In: Dicionário da Educação Profissional em Saúde. Disponível em: http://www.sites.epsjv.fiocruz.br/dicionario/verbetes/intsau.html. Acesso em: 19 de Abr. 2020.

23. ROSA DOA, et al. Violência provocada pelo parceiro íntimo entre usuárias da Atenção Primária à Saúde: prevalência e fatores associados. Saúde debate, Rio de Janeiro, 2018; 42(4): 67-80.

24. SANTOS WJ, et al. Violência Doméstica Contra a Mulher Perpetrada por Parceiro Íntimo: Representações Sociais de Profissionais da Atenção Primária à Saúde. Revista de Pesquisa: Cuidado é Fundamental Online, 2014; 10(3): 770777.

25. SOUZA TMC e MARTINS TF. Vivências de policiais de uma DEAM no Sudoeste Goiano. Fractal, Rev. Psicol. Rio de Janeiro, 2021; 33(1): 21-30.

26. TORRE DNPD, et al. Violência por parceiro íntimo: uma análise sobre usuárias da atenção primária à saúde de Ribeirão das Neves - MG. Rev Med de Minas Gerais, Belo Horizonte, 2005; 26(8): 336-339.

27. UNODC. Escritório das Nações Unidas sobre Drogas e Crime. Handbook on Restorative Justice Programmes. 2. ed. UNODC: Viena, 2020. Disponível em: https://www.unodc.org/documents/justice-and-prison-reform/2001146_Handbook_on_Restorative_Justice_Programmes.pdf Acesso em: 16 jul. 2021. 